\title{
Histological assessment of chronic pancreatitis at necropsy
}

\author{
M Shimizu, M Hirokawa, T Manabe
}

\begin{abstract}
Aims-To evaluate the histological criteria used to diagnose chronic pancreatitis; and to assess interobserver variation among general pathologists.

Methods-Forty five cases of chronic pancreatitis diagnosed in necropsy were reviewed to determine whether the diagnosis was acceptable retrospectively. These cases were diagnosed initially as

0.18 to $66 \%$ in necropsy series. ${ }^{5-7}$ Some pathologists use the term chronic interstitial pancreatitis. ${ }^{5}$ However, according to the 1988 Marseille-Rome classification of pancreatitis, ${ }^{23}$ irregular fibrosis and destruction of exocrine parenchyma are recognised morphological characteristics of chronic pancreatitis. Here, we report a retrospective review of 45 cases of chronic pancreatitis diagnosed at necropsy to determine whether the diagnosis was valid.
\end{abstract} chronic pancreatitis in the final necropsy report complied by general pathologists. In reviewing these cases, special attention was paid to irregular fibrosis and destruction of the lobular architecture.

Results-The 45 cases were re-assigned to seven different diagnostic categories: chronic pancreatitis, 21 (47\%) cases; interstitial fibrosis with or without chronic inflammation, 11 (24\%) cases; repair stage of acute pancreatitis, four (9\%) cases; severe fatty infiltration, three $(7 \%)$ cases; chronic inflammation without interstitial fibrosis, two (4\%) cases; haemochromatosis, one (2\%) case; and undetermined, three $(7 \%)$ cases.

Conclusions-The histological spectrum of chronic pancreatitis was very wide and it was often misdiagnosed. Acinar atrophy, acinar dilation and intralobular fibrosis were diagnostic of chronic pancreatitis. Differential diagnoses include the repair stage of acute pancreatitis, severe fatty infiltration and haemochromatosis. Recognition of these findings may help to reduce overdiagnosis of chronic pancreatitis.

(f Clin Pathol 1996;49:913-915)

Keywords: chronic pancreatitis, necropsy.

Traditionally, chronic pancreatitis was defined as persistent pancreatic damage, both morphological and functional.' Currently, chronic pancreatitis is defined as the presence of chronic inflammatory lesions characterised by destruction of exocrine parenchyma and fibrosis and, at least in the later stages, destruction of endocrine parenchyma. ${ }^{23}$ Chronic pancreatitis is also subclassified into two major forms: chronic calcifying pancreatitis and chronic obstructive pancreatitis. However, chronic inflammatory pancreatitis, characterised by loss of exocrine parenchyma which is replaced by a dense fibrosis with mononuclear cell infiltration, is a separate entity. ${ }^{23}$

Chronic pancreatitis is sometimes diagnosed clinically ${ }^{4}$ and its incidence varies widely from

\section{Methods}

Necropsy records from 1986 to 1990 were reviewed, and $45(5.0 \%)$ cases (27 men and 18 women; mean (range) age 60.4 (17-82) years) of chronic pancreatitis were identified out of a total of 901 necropsy cases. These cases were diagnosed originally by five general pathologists. We evaluated these 45 cases using the 1988 Marseille-Rome classification of pancreatitis. $^{23}$ Therefore, in reviewing these cases, we paid specific attention to irregular fibrosis as well as destruction of lobular architecture. The degree of the lesion was also evaluated, as reported previously. ${ }^{8}$ The term interstitial fibrosis was used for fibrosis confined mainly to the interlobular areas - that is, a mild degree of interstitial fibrosis. ${ }^{8}$ Other features, such as non-papillary or papillary hyperplasia, squamous metaplasia, accumulation of small ducts, duct ectasia, and groups of islets, were also looked for. Here, the term fatty infiltration encompasses stromal infiltration of fat, lipomatosis, ${ }^{9}$ and fatty replacement. The $\chi^{2}$ test and Fisher's exact test were used to analyse the results.

\section{Results}

On review, the 45 cases were assigned to one of seven diagnostic categories: chronic pancreatitis, $21(47 \%)$ cases; interstitial fibrosis with or without chronic inflammation, 11 (24\%) cases; repair stage of acute pancreatitis, four (9\%) cases; moderate to severe fatty infiltration, three $(7 \%)$ cases; chronic inflammation without interstitial fibrosis, two (4\%) cases; haemochromatosis, one $(2 \%)$ case; undetermined, three $(7 \%)$ cases. Of the 21 cases diagnosed as

Table 1 Relation between fibrosis and chronic inflammation in confirmed cases of chronic pancreatitis

\begin{tabular}{lllll}
\hline \multirow{2}{*}{ Fibrosis } & \multicolumn{3}{l}{ Chronic inflammation } \\
\cline { 2 - 5 } & None & Mild & Moderate & Severe \\
\hline Mild & 0 & 6 & 4 & 0 \\
Moderate & 1 & 3 & 4 & 1 \\
Severe & 0 & 1 & 1 & 0 \\
\hline
\end{tabular}


Table 2 Histological comparison between chronic pancreatitis and interstitial fibrosis

\begin{tabular}{lcl}
\hline Associated findings & $\begin{array}{l}\text { Chronic pancreatitis } \\
(21 \text { cases })\end{array}$ & $\begin{array}{l}\text { Interstitial fibrosis } \\
(11 \text { cases) }\end{array}$ \\
\hline Acinar atrophy and dilation & $15(71 \%)$ & $2(18 \%)^{\star \star \star \star}$ \\
Intralobular fibrosis & $18(86 \%)$ & $3(27 \%)^{\star \star \star \star}$ \\
Non-papillary hyperplasia & $11(52 \%)$ & $6(55 \%)$ \\
Papillary hyperplasia & $7(33 \%)$ & $6(55 \%)$ \\
Squamous metaplasia & $8(38 \%)$ & $1(9 \%)$ \\
Accumulation of small ducts & $11(52 \%)$ & $5(45 \%)$ \\
Duct ectasia & $8(38 \%)$ & $2(18 \%)$ \\
Retention cyst & $2(10 \%)$ & $1(9 \%)$ \\
Groups of islets & $11(52 \%)$ & $7(64 \%)$ \\
\hline
\end{tabular}

${ }^{\star} \mathrm{p}<0.05 ;{ }^{\star \star} \mathrm{p}<0.01$.

Table 3 Histological comparison between exacerbated and non-exacerbated chronic pancreatitis

\begin{tabular}{lll}
\hline & $\begin{array}{l}\text { Exacerbated } \\
(n=4)\end{array}$ & $\begin{array}{l}\text { Non-exacerbated } \\
(n=17)\end{array}$ \\
\hline $\begin{array}{c}\text { Acinar dilation } \\
\text { (moderate to severe) }\end{array}$ & $2(50 \%)$ & $2(12 \%)$ \\
$\begin{array}{c}\text { Inspissated secretion } \\
\text { (moderate to severe) }\end{array}$ & $2(50 \%)$ & $2(12 \%)$ \\
$\begin{array}{c}\text { Duct ectasia } \\
\text { Fat necrosis } \\
\text { (moderate to severe) }\end{array}$ & $3(75 \%)$ & $5(29 \%)$ \\
$\begin{array}{c}\text { Chronic inflammation } \\
\text { (moderate to severe) }\end{array}$ & $4(100 \%)$ & $2(12 \%)$ \\
\hline
\end{tabular}

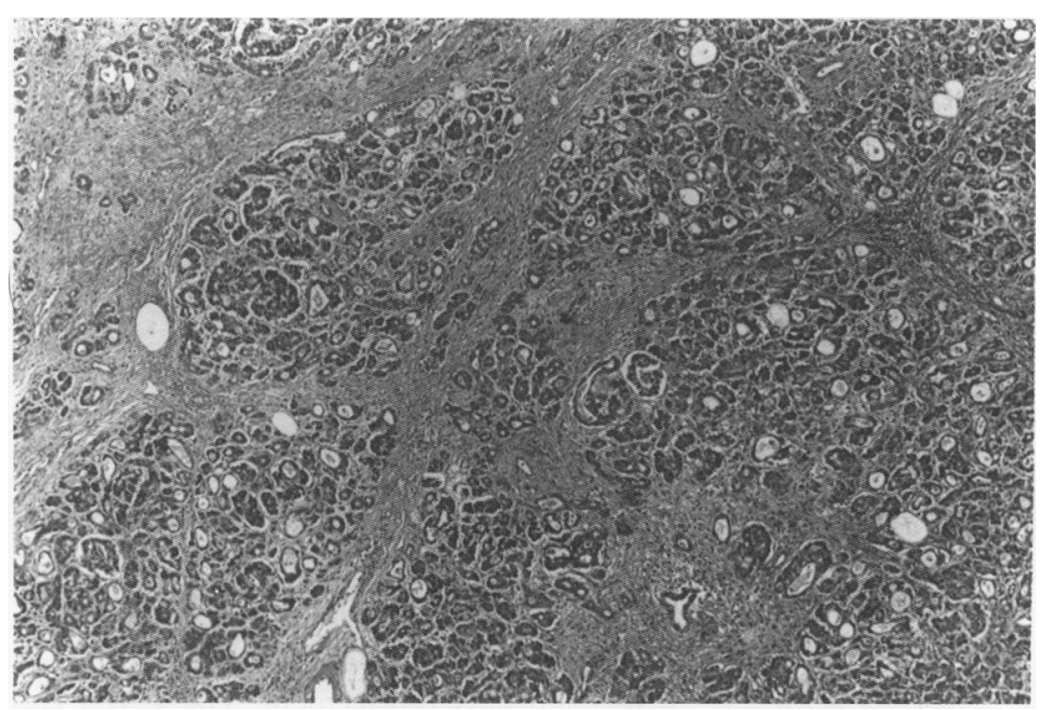

Figure 1 Chronic pancreatitis showing a moderate degree of interstitial fibrosis and a mild degree of chronic inflammation. Acinar atrophy, acinar dilation, and intralobular fibrosis are present.

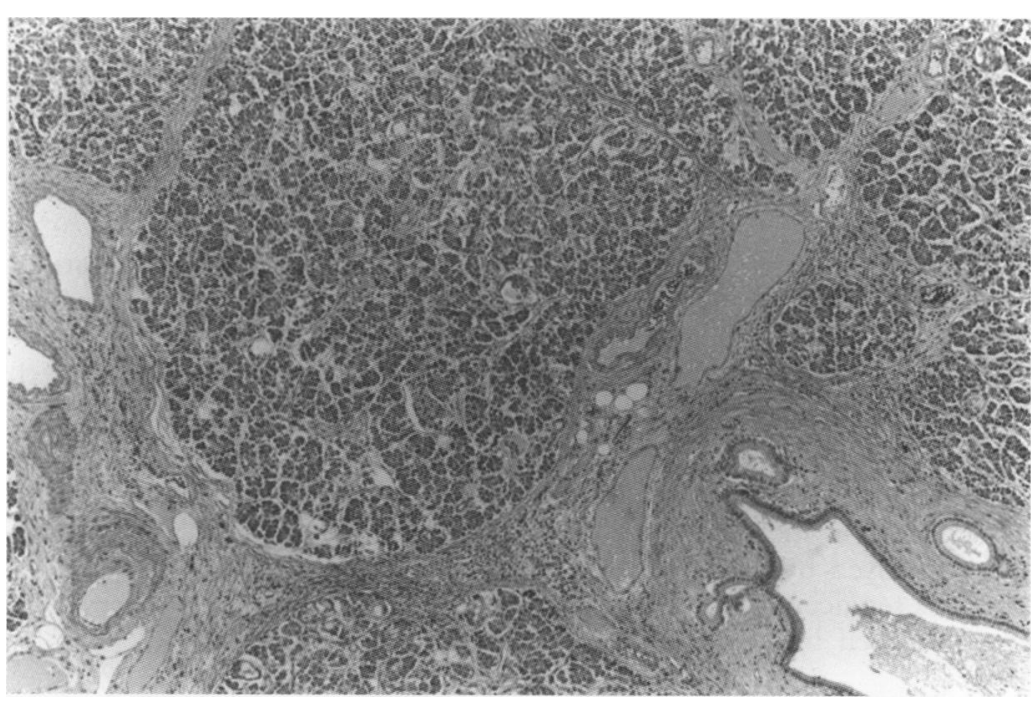

Figure 2 Interstitial fibrosis. A mild degree of interstitial fibrosis and chronic inflammation are present. chronic pancreatitis on review, 15 were men and six were women with a mean (range) age of $63.9(43-82)$ years.

Table 1 shows the relation between fibrosis and chronic inflammation in the 21 cases confirmed as having chronic pancreatitis on review. Fifteen $(71 \%)$ had at least a moderate degree of either fibrosis or chronic inflammation (fig 1). Of the cases of interstitial fibrosis, inflammation was not found in three, while eight had a mild degree of chronic inflammation (fig 2). The histological findings in cases of chronic pancreatitis and interstitial fibrosis are shown in table 2. Acinar atrophy, acinar dilation and intralobular fibrosis were the most useful histological features for differentiating between these two diagnostic categories.

In the 21 cases of confirmed chronic pancreatitis, with the exception of the case without inflammation, two main histological types were noted. The first comprised a mononuclear cell infiltrate, mainly composed of lymphocytes. In the second, the mononuclear cell infiltrate was accompanied by a polymorphonuclear cell infiltrate, mainly composed of neutrophils (fig 3). The latter histological subtype was observed in four cases - that is, they had acute exacerbation of chronic pancreatitis. The differences between these two histological types are shown in table 3 . The case without inflammation was included in the former type. In cases of exacerbation of chronic pancreatitis, duct ectasia, fat necrosis, acinar dilation, and inspissated secretion were often observed.

Significant fibrosis was not observed in cases of acute pancreatitis, fatty infiltration and haemochromatosis (figs 4 and 5).

A definitive diagnosis could not be reached in three cases. The differential diagnoses were: exacerbation of chronic pancreatitis versus repair stage of acute pancreatitis in a 35 year old man; pancreatic cancer with chronic pancreatitis versus fibrosis secondary to pancreatic cancer in a 66 year old woman; and chronic pancreatitis versus interstitial fibrosis with chronic inflammation in a 72 year old man.

\section{Discussion}

Our study revealed that the histological spectrum of chronic pancreatitis was very wide according to general pathologists. It was often misdiagnosed because chronic pancreatitis may be diagnosed by some general pathologists by the presence of chronic inflammation or interstitial fibrosis. As the morphology of chronic pancreatitis is characterised by irregular fibrosis with destruction and permanent loss of exocrine parenchyma, ${ }^{23}$ acinar atrophy, acinar dilation and intralobular fibrosis are important histological features. In addition, the degree of chronic inflammation and interstitial fibrosis should be considered. In our study, a mild degree of interstitial fibrosis was separated from interstitial fibrosis in general as shown in Methods because, in the latest international meeting on the classification of pancreatitis, perilobular fibrosis, which is sometimes associated with some degree of intralobular fibrosis but without evident loss of 


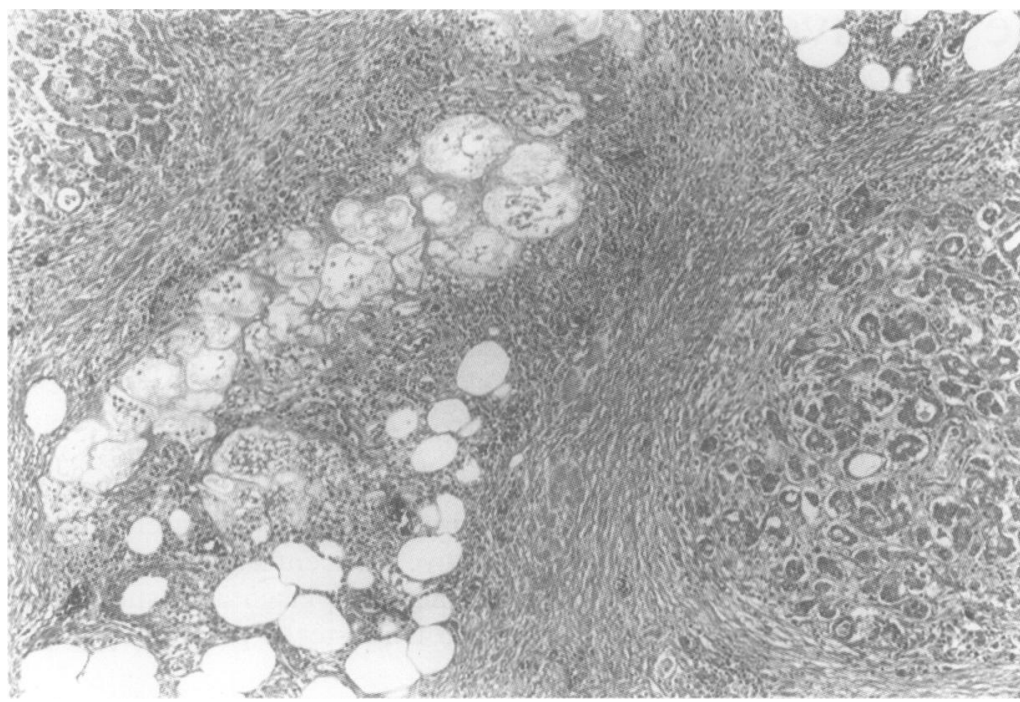

Figure 3 Exacerbation of chronic pancreatitis. Fat necrosis with neutrophilic infiltration can be seen.

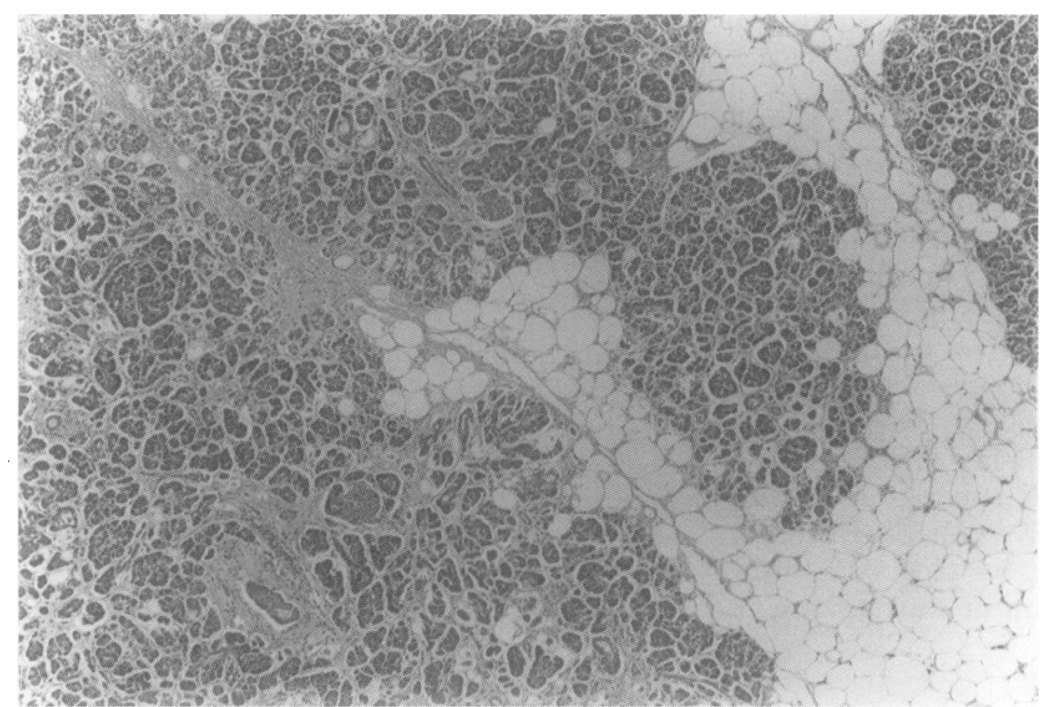

Figure 4 Fatty infiltration.

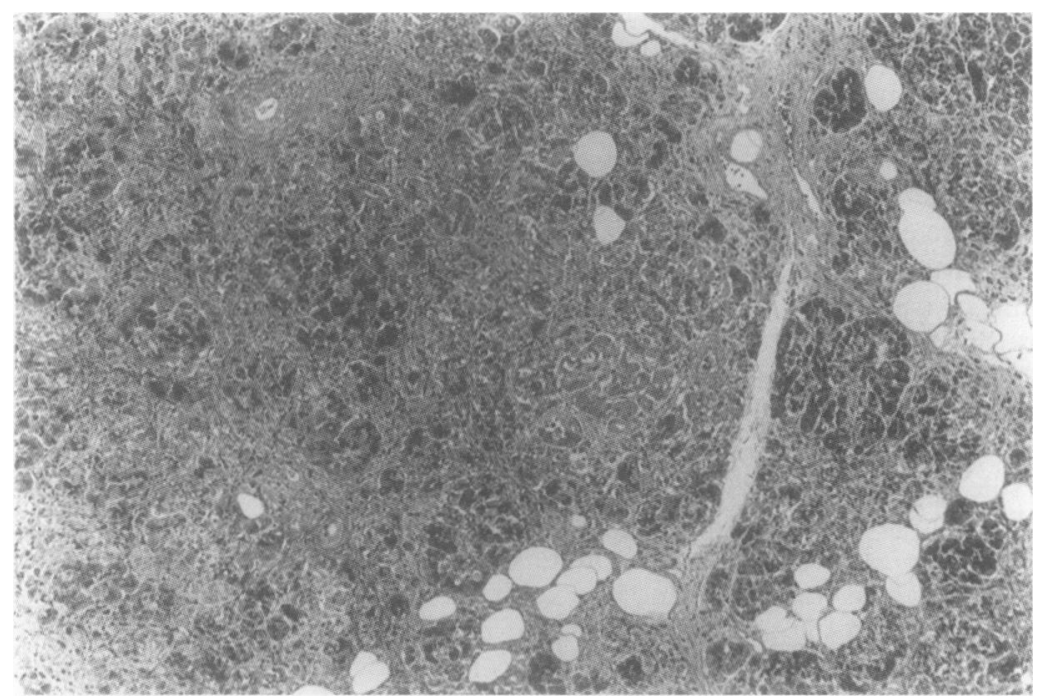

Figure 5 Haemochromatosis. Deposition of haemosiderin can be seen, but destruction of lobular architecture is lacking.
As differential diagnoses, the repair stage of acute pancreatitis, interstitial fibrosis, haemochromatosis, and severe fatty infiltration, should be always kept in mind. Recognition of these differential diagnoses may be useful for avoiding misdiagnosis of chronic pancreatitis by general pathologists. In acute pancreatitis no to a mild degree of interstitial fibrosis, immature fibroblasts and significant oedema are key features. The most prominent feature of exacerbated chronic pancreatitis, however, is acute inflammatory change, mainly affecting the pancreatic ducts.

Irregular fibrosis and fibrosis localised between pancreatic and fatty tissue are observed in chronic pancreatitis, but not in fatty infiltration alone. Lipomatosis is also said to be related to increasing age. ${ }^{9}$ In fact, the mean (range) age of cases of fatty infiltration in our study was 80 (77-82) years, which is much older than that of cases of chronic pancreatitis. Haemochromatosis may resemble chronic pancreatitis histologically as it is one of the aetiological factors of chronic pancreatitis. ${ }^{1}$ However, the presence of fibrosis alone without destruction of pancreatic parenchyma is not indicative of chronic pancreatitis unless irregular fibrosis and lobular destruction are also present. $^{23}$

Duct lesions including dilation, inspissated secretions, abnormal epithelium, and calculi have been described as components of chronic pancreatitis. ${ }^{10}$ However, there were no significant differences in the ductal changes observed in the present study between chronic pancreatitis and interstitial fibrosis.

In conclusion, the histological spectrum of chronic pancreatitis was very wide and it was often misdiagnosed. Acinar atrophy, acinar dilation and intralobular fibrosis were diagnostic of chronic pancreatitis. Differential diagnoses include the repair stage of acute pancreatitis, severe fatty infiltration and haemochromatosis. Recognition of these findings may help to reduce overdiagnosis of chronic pancreatitis.

1 Klöppel G, Heitz PU. Pancreatitis. In: Pancreatic pathology. Edinburgh: Churchill Livingstone, 1984:44-72.

2 Chari ST, Singer MV. The problem of classification and staging of chronic pancreatitis. Proposals based on current knowledge of its natural history. Scand $\mathcal{F}$ Gastroenterol 1994;29:949-60.

3 Sarles H, Adler G, Dani R, Frey C, Gullo L, Harada H, et al. Classification of pancreatitis and definition of pancreatic Classification of pancreatitis and
diseases. Digestion 1989;43:234-6.

4 Bozkurt T, Braun U, Leferink S, Gilly G, Lux G, Comparison of pancreatic morphology and exocrine functional impairment in patients with chronic pancreatitis. Gut 1994;35:1132-6

5 Czernobilsky B, Mikat KW. The diagnostic significance of interstitial pancreatitis found at autopsy. Am $\mathcal{Y}$ Clin Pathol 1964;41:33-43.

6 Edmondson HA, Bullock WK, Mehl JW. Chronic pancreatitis and lithiasis. I. A clinicopathologic study of 62 cases of chronic pancreatitis. Am $\mathcal{f}$ Pathol 1949;25:1227-47.

7 Stein AA, Powers SR Jr. Terminal pancreatitis. Arch Pathol 1958;65:445-8.

8 Shimizu M, Hayashi T, Saitoh Y, Itoh $H$. Interstitial fibrosis in the pancreas. Am $\mathcal{F}$ Clin Pathol 1989;91:531-4. 9 Olsen TS. Lipomatosis of the pancreas in autopsy material and its relation to age and over
Scand $[A]$ 1978;86:367-73.

exocrine parenchyma, was not regarded as chronic pancreatitis by most participants. ${ }^{3}$ Surg Pathol 1989;13(Suppl 1):50-65. 